\title{
Soft and Coulomb gluon resummation in squark-antisquark production at the LHC
}

\author{
M. Beneke \\ Institut für Theoretische Physik E, RWTH Aachen University, \\ D - 52056 Aachen, Germany

\section{P. Falgari*i} \\ IPPP, Department of Physics, University of Durham, \\ Durham DH1 3LE, England \\ E-mail: pietro.falgari@durham.ac.uk

\section{Schwinn} \\ Albert-Ludwigs Universität Freiburg, \\ Physikalisches Institut, D-79104 Freiburg, Germany
}

\begin{abstract}
We present results for a combined resummation of soft and Coloumb gluon corrections in squarkantisquark production at the LHC, including the non-trivial interference of the two effects and the contribution of squark-antisquark bound states below threshold. The total correction to the nextto-leading order approximation is found to be sizeable, and amounts to $5-14 \%$ in the squark mass region $200 \mathrm{GeV}-2 \mathrm{TeV}$. The scale dependence of the total cross section is also reduced.
\end{abstract}

RADCOR 2009 - 9th International Symposium on Radiative Corrections (Applications of Quantum Field Theory to Phenomenology)

October 25-30 2009

Ascona, Switzerland

\footnotetext{
* Speaker.

†Preprint TTK-10-12, SFB/CPP-10-14, IPPP/10/04, DCPT/10/08, FR-PHENO-2010-007. The work of M.B. is supported by the DFG Sonderforschungsbereich/Transregio 9 "Computergestützte Theoretische Teilchenphysik".
} 


\section{Introduction}

The total cross sections for the processes $p p^{\prime} \rightarrow H H^{\prime}+X$, where $p p^{\prime} \in\{q q, q \bar{q}, g g, g q, g \bar{q}\}$ and $H, H^{\prime}$ are heavy particles in arbitrary representations $R, R^{\prime}$ of the $S U(3)$ colour algebra (top quarks, squarks, gluinos...), contain classes of contributions which are enhanced near the partonic threshold region $\beta \equiv \sqrt{1-4 M^{2} / \hat{s}} \rightarrow 0$, with $M=\left(M_{H}+M_{H^{\prime}}\right) / 2$ the average mass of the two heavy particles and $\hat{s}$ the partonic centre-of-mass energy. These corrections arise from soft-gluon radiation off initial- and final-state particles $\left(\sim \alpha_{s}^{n} \ln ^{m} \beta\right.$, "threshold logaritms") and exchange of Coulomb gluons between the two non-relativistic heavy particles $\left(\sim\left(\alpha_{s} / \beta\right)^{n}\right.$, "Coulomb singularities"), and should be resummed to all orders in $\alpha_{s}$ if the hadronic cross section is dominated by the partonic threshold region. Resummation of threshold logarithms in the Mellin-moment space formalism has been discussed, for example, in [1,2], and studies of the effects of Coulomb resummation in production of top quarks and SUSY particles have been presented in $[3,4,5]$. However, the issue of factorisation and simultaneous resummation of soft and Coulomb effects in pair production has been addressed rigorously only recently in [6], employing effective-theory techniques similar to ones previously applied in the context of DIS and Drell-Yan [7, 8]. Here we present the application of results obtained in [6] to the process of squark-antisquark production at the LHC, and discuss the numerical relevance of soft and Coulomb resummation for theoretical predictions of the total cross section (see also [9]).

\section{Factorisation and RG evolution equations}

In [6] it has been shown that near the partonic threshold $\hat{s} \sim 4 M^{2}$ the total partonic cross section for the pair-production process factorises, at leading-order in $\beta$ and for particles produced in an $S$-wave state, according to

$$
\hat{\sigma}_{p p^{\prime}}\left(\beta, \mu_{f}\right)=\sum_{i, i^{\prime}} H_{i i^{\prime}}\left(M, \mu_{f}\right) \int d \omega \sum_{R_{\alpha}} J_{R_{\alpha}}\left(E-\frac{\omega}{2}\right) W_{i i^{\prime}}^{R_{\alpha}}\left(\omega, \mu_{f}\right),
$$

with $E=\sqrt{\hat{s}}-2 M$. Here $H_{i i^{\prime}}\left(M, \mu_{f}\right)$ denotes a process-dependent hard coefficient encoding short-distance effects, decomposed over a basis of colour-state operators $\left\{c_{\{a\}}^{(i)}\right\}$. The soft function $W_{i i^{\prime}}^{R_{\alpha}}\left(\omega, \mu_{f}\right)$ is defined in terms of matrix elements of soft Wilson lines, decomposed over the basis $\left\{c_{\{a\}}^{(i)}\right\}$ and projected onto the irreducible colour representations $R_{\alpha}$ of the $H H^{\prime}$ system, $R \otimes R^{\prime}=\sum_{\alpha} R_{\alpha}$. The function $J_{R_{\alpha}}$ describes the internal evolution of the heavy-particle pair $H H^{\prime}$, which is driven by Coulomb-gluon exchange. Near the partonic threshold the basis $\left\{c_{\{a\}}^{(i)}\right\}$ can be chosen such that the soft function $W_{i i^{\prime}}^{R_{\alpha}}\left(\omega, \mu_{f}\right)$ is diagonal in colour space to all orders in $\alpha_{s}$, and the colour structure of (2.1) simplifies significantly [6].

The resummation of threshold logarithms follows the formalism of [7], and is a generalisation of the Drell-Yan case [8] to arbitrary colour representations $R_{\alpha}$ of the heavy-particle pair system. From results on the IR structure of general massive amplitudes in QCD [10] it follows that the diagonal elements of the hard function $H_{i}\left(M, \mu_{f}\right) \equiv H_{i i}\left(M, \mu_{f}\right)$ satisfy a renormalisation-group evolution equation given by [6]

$$
\frac{d}{d \ln \mu_{f}} H_{i}\left(M, \mu_{f}\right)=\left(\gamma_{\text {cusp }}\left(C_{r}+C_{r^{\prime}}\right) \ln \left(\frac{4 M^{2}}{\mu_{f}^{2}}\right)+2 \gamma_{i}^{V}\right),
$$




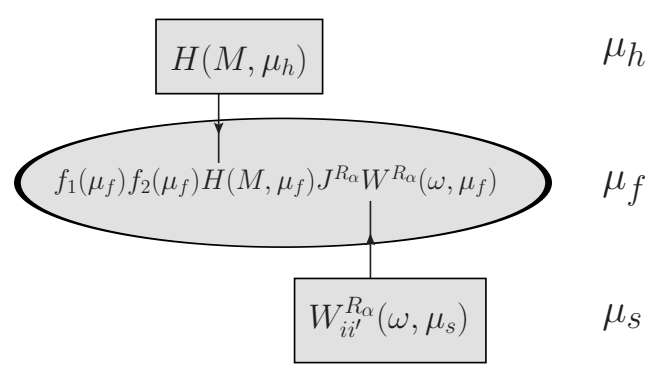

Figure 1: Schematic representation of the resummation of threshold logarithms via RGEs.

where $\gamma_{\text {cusp }}$ denotes the cusp anomalous dimension, $C_{r}$ and $C_{r^{\prime}}$ are the Casimir invariants of the initial-state representations, and $\gamma_{i}^{V}$ can be written in terms of single-particle anomalous dimensions $\gamma_{i}^{V}=\gamma^{r}+\gamma^{r^{\prime}}+\gamma_{H, s}^{R_{\alpha}}$. From the scale-invariance of the total hadronic cross section, one can derive an analogous evolution equation for the soft function $W_{i}^{R_{\alpha}}\left(\omega, \mu_{f}\right)$ [8],

$$
\begin{aligned}
\frac{d}{d \ln \mu_{f}} W_{i}^{R_{\alpha}}\left(\omega, \mu_{f}\right)= & -2\left[\left(C_{r}+C_{r^{\prime}}\right) \gamma_{\text {cusp }} \ln \left(\frac{\omega}{\mu_{f}}\right)+2 \gamma_{W, i}^{R_{\alpha}}\right] W_{i}^{R_{\alpha}}\left(\omega, \mu_{f}\right) \\
& -2\left(C_{r}+C_{r^{\prime}}\right) \gamma_{\text {cusp }} \int_{0}^{\omega} d \omega^{\prime} \frac{W_{i}^{R_{\alpha}}\left(\omega^{\prime}, \mu_{f}\right)-W_{i}^{R_{\alpha}}\left(\omega, \mu_{f}\right)}{\omega-\omega^{\prime}},
\end{aligned}
$$

with the anomalous dimension $\gamma_{W, i}^{R_{\alpha}}$ given by $\gamma_{W, i}^{R_{\alpha}}=\gamma_{i}^{V}+\gamma^{\phi, r}+\gamma^{\phi, r^{\prime}}$, and $\gamma^{\phi, r}$ defined by the large- $x$ limit of the Altarelli-Parisi splitting function

$$
P_{p / k}\left(x, \mu_{f}\right)=\left(2 C_{r} \gamma_{\operatorname{cusp}} \frac{1}{[1-x]_{+}}+2 \gamma^{\phi, r} \delta(1-x)\right) \delta_{p k}+\ldots
$$

\section{Momentum-space resummation}

In the approach of [7] the resummation of threshold logarithms is obtained directly in momentum space by calculating the hard and soft functions at scales $\mu_{h}$ and $\mu_{s}$ respectively, and evolving them to the common factorisation scale $\mu_{f}$ using the RG equations (2.2) and (2.3), as schematically depicted in figure 1 . The hard scale $\mu_{h}$ and soft scale $\mu_{s}$ are chosen to minimise radiative corrections to $H_{i}\left(M, \mu_{h}\right)$ and $W_{i}^{R_{\alpha}}\left(\omega, \mu_{s}\right)$. As anticipated above, the solutions of equations (2.2) and (2.3) are generalisations of the Drell-Yan case discussed in [8] and read

$$
\begin{aligned}
H_{i}^{\mathrm{res}}\left(M, \mu_{f}\right) & =\exp \left[4 S\left(\mu_{h}, \mu_{f}\right)-2 a_{i}^{V}\left(\mu_{h}, \mu_{f}\right)\right]\left(\frac{4 M^{2}}{\mu_{h}^{2}}\right)^{-2 a_{\Gamma}\left(\mu_{h}, \mu_{f}\right)} H_{i}\left(M, \mu_{h}\right), \\
W_{i}^{R_{\alpha} \text {,res }}\left(\omega, \mu_{f}\right) & =\exp \left[-4 S\left(\mu_{s}, \mu_{f}\right)+2 a_{W, i}^{R_{\alpha}}\left(\mu_{s}, \mu_{f}\right)\right] \tilde{s}_{i}^{R_{\alpha}}\left(\partial_{\eta}, \mu_{s}\right) \frac{1}{\omega}\left(\frac{\omega}{\mu_{s}}\right)^{2 \eta} \theta(\omega) \frac{e^{-2 \gamma_{E} \eta}}{\Gamma(2 \eta)},(3.1
\end{aligned}
$$

where

$$
\begin{aligned}
& S(v, \mu)=-\left(C_{r}+C_{r^{\prime}}\right) \int_{\alpha_{s}(v)}^{\alpha_{s}(\mu)} d \alpha_{s} \frac{\gamma_{\text {cusp }}\left(\alpha_{s}\right)}{2 \beta\left(\alpha_{s}\right)} \int_{\alpha_{s}(v)}^{\alpha_{s}} \frac{d \alpha_{s}^{\prime}}{\beta\left(\alpha_{s}^{\prime}\right)}, \\
& a_{\Gamma}(v, \mu)=-\left(C_{r}+C_{r^{\prime}}\right) \int_{\alpha_{s}(v)}^{\alpha_{s}(\mu)} d \alpha_{s} \frac{\gamma_{\text {cusp }}\left(\alpha_{s}\right)}{2 \beta\left(\alpha_{s}\right)}, \quad a_{i}^{X}(v, \mu)=-\int_{\alpha_{s}(v)}^{\alpha_{s}(\mu)} d \alpha_{s} \frac{\gamma_{i}^{X}\left(\alpha_{s}\right)}{\beta\left(\alpha_{s}\right)},
\end{aligned}
$$


and $\hat{s}_{i}^{R_{\alpha}}\left(\rho, \mu_{s}\right)$ is the Laplace-transform of $W_{i}^{R_{\alpha}}\left(\omega, \mu_{s}\right)$ with respect to the variable $s=1 /\left(e^{\gamma_{E}} \mu e^{\rho / 2}\right)$,

$$
\hat{s}_{i}^{R_{\alpha}}\left(\rho, \mu_{s}\right)=\int_{0_{-}}^{\infty} d \omega e^{-s \omega} W_{i}^{R_{\alpha}}\left(\omega, \mu_{s}\right) .
$$

The one-loop expression for $\tilde{s}_{i}^{R_{\alpha}}\left(\partial_{\eta}, \mu_{s}\right)$ for arbitrary colour representations was computed in [6]. Notice that the resummed hard and soft functions in eq. (3.1) are formally independent of the choice of $\mu_{h}$ and $\mu_{s}$, but when $H\left(M, \mu_{h}\right)$ and $\hat{s}_{i}^{R_{\alpha}}\left(\partial_{\eta}, \mu_{s}\right)$ are truncated at a finite perturbative order a dependence on the hard and soft scales is introduced, which is however of higher order in $\alpha_{s}$.

The resummation of the velocity-enhanced terms $\left(\alpha_{s} / \beta\right)^{n}$ associated with Coulomb-gluon exchange is obtained by relating the potential function $J_{R_{\alpha}}$ to the zero-distance Coulomb Green function, $J_{R_{\alpha}}=2 \operatorname{Im} G_{C, R_{\alpha}}^{(0)}(0,0 ; E)$. Using the representation provided in [11], this gives

$$
\begin{aligned}
J_{R_{\alpha}}(E)= & -\frac{\left(2 m_{\mathrm{red}}\right)^{2}}{2 \pi} \operatorname{Im}\left\{\sqrt{-\frac{E}{2 m_{\mathrm{red}}}}+\alpha_{s}\left(-D_{R_{\alpha}}\right)\left[\frac{1}{2} \ln \left(-\frac{8 m_{\mathrm{red}} E}{\mu_{f}^{2}}\right)\right.\right. \\
& \left.\left.-\frac{1}{2}+\gamma_{E}+\psi\left(1-\frac{\alpha_{s}\left(-D_{R_{\alpha}}\right)}{2 \sqrt{-E /\left(2 m_{\mathrm{red}}\right)}}\right)\right]\right\},
\end{aligned}
$$

with $D_{R_{\alpha}}=\left(C_{R_{\alpha}}-C_{R}-C_{R^{\prime}}\right) / 2$ and $m_{\text {red }}=M_{H} M_{H^{\prime}} /\left(M_{H}+M_{H^{\prime}}\right)$. The second term on the first line of (3.4) encodes the contribution to the cross section from a single Coulomb-exchange diagram, while the second line accounts for the contribution of two or more gluons. Beside modifying the shape of the cross section above threshold $(E>0)$, the resummation of Coulomb effects leads to the appearance of heavy-particle bound states below threshold for an attractive Coulomb potential $\left(D_{R_{\alpha}}<0\right)$. In the limit of vanishing width, $\Gamma_{H}=\Gamma_{H^{\prime}}=0$, and for $E<0, J_{R_{\alpha}}$ reduces to

$$
J_{R_{\alpha}}(E)=2 \sum_{n=1}^{\infty}\left(\frac{m_{\tilde{q}} \alpha_{s}\left(-D_{R_{\alpha}}\right)}{2 n}\right)^{3} \delta\left(E+m_{\tilde{q}}\left(\frac{\alpha_{s}\left(-D_{R_{\alpha}}\right)}{2 n}\right)^{2}\right) \theta\left(-D_{R_{\alpha}}\right) .
$$

\section{Threshold resummation for squark-antisquark production}

We now apply the results presented in the previous section to the NLL soft resummation and Coulomb resummation of the squark-antisquark total production cross section. The resummed cross section for the partonic channel $p p^{\prime}$ is

$$
\hat{\sigma}_{p p^{\prime}}^{\mathrm{res}}\left(\hat{s}, \mu_{f}\right)=\sum_{i} H_{i}^{\mathrm{NLL}}\left(m_{\tilde{q}}, \mu_{f}\right) \int d \omega \sum_{R_{\alpha}} J_{R_{\alpha}}\left(E-\frac{\omega}{2}\right) W_{i}^{R_{\alpha}, \mathrm{NLL}}\left(\omega, \mu_{f}\right) .
$$

The NLL resummed hard and soft functions in (4.1) are given by the expressions in eq. (3.1), where the cusp anomalous dimension $\gamma_{\text {cusp }}$ is included at the two-loop accuracy, the anomalous dimensions $\gamma_{i}^{V}, \gamma_{W, i}^{R_{\alpha}}$ at one-loop accuracy, and $H_{i}\left(M, \mu_{h}\right)$ and $\hat{s}_{i}^{R_{\alpha}}\left(\partial_{\eta}, \mu_{s}\right)$ are replaced by their tree-level expressions $H_{i}^{(0)}\left(M, \mu_{h}\right)$ and $\hat{s}_{i}^{R_{\alpha}(0)}\left(\partial_{\eta}, \mu_{s}\right)=1$ (for more details on the reorganised perturbative expansion in presence of soft and Coulomb resummation we refer to [6]). The resummed cross section (4.1) is then matched onto the full fixed-order NLO result

$$
\hat{\sigma}_{p p^{\prime}}^{\mathrm{match}}\left(\hat{s}, \mu_{f}\right)=\left[\hat{\sigma}_{p p^{\prime}}^{\mathrm{res}}\left(\hat{s}, \mu_{f}\right)-\left.\hat{\sigma}_{p p^{\prime}}^{\mathrm{res}}\left(\hat{s}, \mu_{f}\right)\right|_{\mathrm{NLO}}\right]+\hat{\sigma}_{p p^{\prime}}^{\mathrm{NLO}}\left(\hat{s}, \mu_{f}\right),
$$


using the parametrisation of the NLO cross section extracted in [12] from numerical results obtained with Prospino [13]. Our final prediction for the hadronic cross section is given by the convolution of (4.2) with the MSTW2008 NLO PDFs. The default value for the soft scale, denoted with $\tilde{\mu}_{s}$, is chosen such that the one-loop soft correction to the hadronic cross section is minimised. This is analogous to the procedure introduced in [8], and yields $\bar{\mu}_{s}=123-455 \mathrm{GeV}$ for the squark mass range $m_{\tilde{q}}=200 \mathrm{GeV}-2 \mathrm{TeV}$. Furthermore, we evaluate the running coupling in $J_{R_{\alpha}}$ at the scale $\mu_{C}=\operatorname{Max}\left\{2 m_{\tilde{q}} \beta, C_{F} m_{\tilde{q}} \alpha_{s}\left(\mu_{C}\right)\right\}$. This choice is motivated by the characteristic virtuality of a Coulomb gluon, $q^{2} \sim m_{\tilde{q}}^{2} \beta^{2}$, and by the inverse Bohr radius of the first $H H^{\prime}$ bound state, $1 / r_{B}=C_{F} m_{\tilde{q}} \alpha_{s} / 2$. Finally, we identify the hard and factorisation scale, $\mu_{h}=\mu_{f}$, and choose the latter of order $m_{\tilde{q}}$. This means that the resummed hard function in (4.1) reduces to its tree-level expression, since the functions (3.2) vanish for this particular scale choice. The function $H_{i}^{(0)}\left(m_{\tilde{q}}, \mu_{f}\right)$ is extracted from the expansion at threshold of the tree-level cross section $\sigma_{i}^{(0)}=\frac{m_{\tilde{q}}}{2 \pi} \beta H_{i}^{(0)}+O\left(\beta^{3}\right)$.

The numerical effects of soft-Coulomb resummation on the total hadronic cross section, normalised to the full NLO result, are shown in figure 2 , for $\sqrt{s}=14 \mathrm{TeV}$, degenerate squark masses, $m_{\tilde{g}} / m_{\tilde{q}}=1.25$ and $\mu_{f}=m_{\tilde{q}}$. The four curves represent different contributions contained in the resummed cross section (4.1). The low-

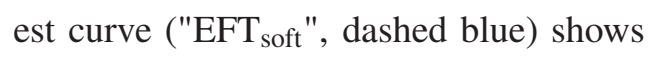
the effect of NLL soft-gluon resummation without any Coulomb effect, corresponding to setting $J_{R_{\alpha}}$ to its tree-level value in (4.1). "EFT soft+C" (dot-dashed red) includes, on top of soft effects, Coulomb

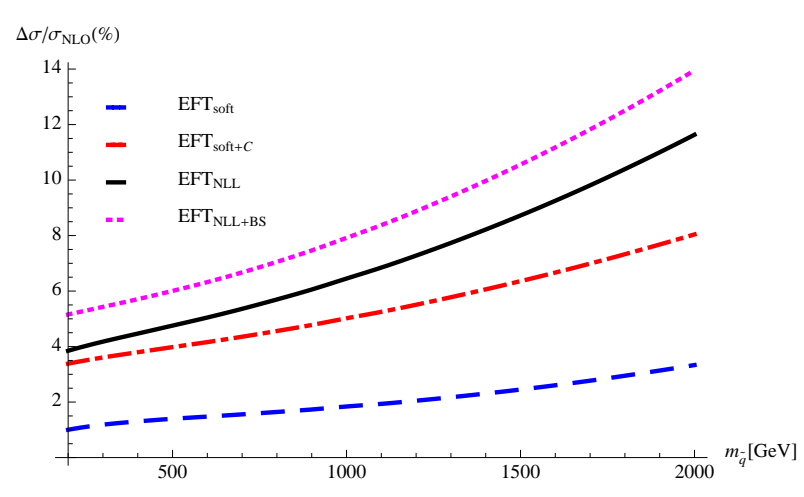

Figure 2: Corrections to the NLO squark-antisquark cross section from soft-Coulomb resummation as functions of the squark mass $m_{\tilde{q}}$. resummation, but no interference of the two. This is partially included in the third curve ("EFT ${ }_{\mathrm{NLL}}$ ", solid black) convoluting the one-loop Coulomb contribution (second term in (3.4)) with the full NLL soft function (see also [9]). Finally, the contribution to the cross section from bound states below threshold is added in the last curve ("EFT $\mathrm{FLL}_{\mathrm{BS}}$ ", dotted magenta). The pure soft resummation is in qualitative agreement with the Mellin-space results quoted in [5], while the choice of a running scale in the Coulomb corrections leads to much larger effects than in [5], where $\alpha_{s}$ at the scale $\mu_{f}$ was used. Also, the soft-Coulomb interference and bound-state contributions shown in figure 2 turn out to be comparable in size to pure soft and Coulomb effects, and are therefore necessary for precise theoretical predictions.

The reduced scale dependence of the resummed cross section is presented in figure 3 . The left plot shows the LO, NLO and resummed result as functions of the factorisation scale $\mu_{f}$, for $m_{\tilde{q}}=1 \mathrm{TeV}$ and $\mu_{h}=\mu_{f}$. The green band represents the uncertainty associated with the choice of the soft scale, which varies in the interval $\bar{\mu}_{s} / 2<\mu_{s}<2 \bar{\mu}_{s}$. The scale dependence is clearly reduced for large $\mu_{f}$, but only mildly improved at small values of the scale, where $\ln \left(\mu_{s} / \mu_{f}\right)=O(1)$ and the effect of soft resummation is negligible. It can be argued that in this region $\left(\mu_{f} \lesssim 0.2 m_{\tilde{q}}\right)$ the identification of the hard scale with the factorisation scale is not justified, and the two scales should 

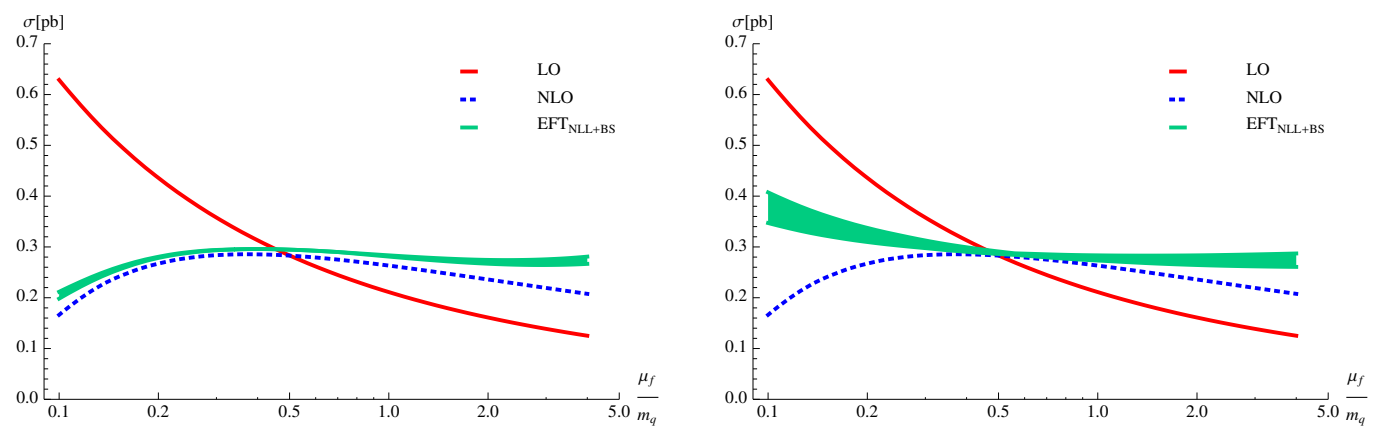

Figure 3: Scale dependence of the LO (solid red), NLO (dotted blue) and resummed cross section (green band) for different choices of $\mu_{s}$ and $\mu_{h}$. See the text for explanation.

be kept separate. This is shown in the right plot in figure 3. In this case the green band is obtained by varying $\mu_{h}$ and $\mu_{s}$, independently from the factorisation scale, in the intervals $m_{\tilde{q}}<\mu_{h}<4 m_{\tilde{q}}$ and $\bar{\mu}_{s} / 2<\mu_{s}<2 \bar{\mu}_{s}$, respectively. While the cross section is not significantly modified with respect to the left plot at large scales, in the region $\mu_{f} \ll m_{\tilde{q}}$ the appearance of large logarithms of $\mu_{h} / \mu_{f}$, and their resummation in $H_{i}^{\mathrm{NLL}}\left(m_{\tilde{q}}, \mu_{f}\right)$, leads to sizeable corrections to the cross section.

\section{References}

[1] N. Kidonakis and G. Sterman, Nucl. Phys. B505, 321 (1997), [hep-ph/9705234].

[2] R. Bonciani, S. Catani, M. L. Mangano and P. Nason, Nucl. Phys. B529, 424 (1998) (Erratum-ibid. B 803 (2008) 234), [hep-ph/9801375].

[3] K. Hagiwara, Y. Sumino and H. Yokoya, Phys. Lett. B 666 (2008) 71 [arXiv:0804.1014 [hep-ph]]; K. Hagiwara and H. Yokoya, JHEP 0910 (2009) 049 [arXiv:0909.3204 [hep-ph]].

[4] Y. Kiyo, J. H. Kuhn, S. Moch, M. Steinhauser and P. Uwer, Eur. Phys. J. C 60 (2009) 375 [arXiv:0812.0919 [hep-ph]].

[5] A. Kulesza and L. Motyka, Phys. Rev. D 80 (2009) 095004 [arXiv:0905.4749 [hep-ph]]; W. Beenakker et al. JHEP 0912 (2009) 041 [arXiv:0909.4418 [hep-ph]].

[6] M. Beneke, P. Falgari and C. Schwinn, Nucl. Phys. B 828 (2010) 69, arXiv:0907.1443 [hep-ph], and work in preparation.

[7] T. Becher, M. Neubert and B. D. Pecjak, JHEP 0701 (2007) 076 [arXiv:hep-ph/0607228].

[8] T. Becher, M. Neubert and G. Xu, JHEP 0807 (2008) 030 [arXiv:0710.0680 [hep-ph]].

[9] M. Beneke, P. Falgari and C. Schwinn, proceedings of 2009 Europhysics Conference on High Energy Physics arXiv:0909.3488 [hep-ph]

[10] T. Becher and M. Neubert, Phys. Rev. D 79 (2009) 125004 [Erratum-ibid. D 80 (2009) 109901] [arXiv:0904.1021 [hep-ph]].

[11] M. Beneke, in: Proceedings of the 8th International Symposium on Heavy Flavour Physics, Southampton, England, 25-29 July 1999, [arXiv:hep-ph/9911490].

[12] U. Langenfeld and S. O. Moch, Phys. Lett. B 675 (2009) 210 [arXiv:0901.0802 [hep-ph]].

[13] W. Beenakker, R. Hopker, M. Spira and P. M. Zerwas, Nucl. Phys. B 492 (1997) 51 [arXiv:hep-ph/9610490]. 